\title{
PAPER \\ Pseudo Eigenbeam-Space Division Multiplexing (PE-SDM) in Frequency-Selective MIMO Channels
}

\author{
Hiroshi NISHIMOTO $^{\dagger a)}$, Student Member, Toshihiko NISHIMURA ${ }^{\dagger b)}$, Takeo OHGANE ${ }^{\dagger c)}$, \\ and Yasutaka OGAWA ${ }^{\dagger \mathrm{d})}$, Members
}

\begin{abstract}
SUMMARY In a frequency-selective multiple-input multiple-output (MIMO) channel, the optimum transmission is achieved by beamforming with eigenvectors obtained at each discrete frequency point, i.e., an extension of eigenbeam-space division multiplexing (E-SDM). However, the calculation load of eigenvalue decomposition at the transmitter increases in proportion to the number of frequency points. In addition, frequencyindependent eigenvectors increase the delay spread of the effective channel observed at the receiver. In this paper, we propose a pseudo eigenvector scheme for the purpose of mitigating the calculation load and maintaining frequency continuity (or decreasing the delay spread). First, we demonstrate that pseudo eigenvectors reduce the delay spread of the effective channels with low computational complexity. Next, the practical performance of the pseudo E-SDM (PE-SDM) transmission is evaluated. The simulation results show that PE-SDM provides almost the same or better performance compared with E-SDM when the receiver employs a time-windowing-based channel estimation available in the low delay spread cases.

key words: MIMO system, frequency-selective fading, transmit beamforming, effective channel, frequency continuity, singular value decomposition (SVD), pseudo eigenvector, pseudo eigenbeam-space division multiplexing $(P E-S D M)$, channel estimation
\end{abstract}

\section{Introduction}

Recently, the multiple-input multiple-output (MIMO) system has been extensively studied because it can meet the growing demand for higher capacity in digital wireless communication fields [1]-[6], and is already the core technology in some wireless standards [7]-[9]. When the MIMO channel state information (CSI) is available at the transmitter, eigenbeam-space division multiplexing (E-SDM) can be applied [10]-[14]. This method enables us to transmit spatially-orthogonal substreams by using eigenvectors, which are obtained by singular value decomposition (SVD) of the MIMO channel matrix, as transmit weight vectors. Therefore, this architecture is also called SVD-MIMO [15] (or closed-loop MIMO [16] since the CSI feedback is generally required). The maximum throughput of the MIMO channel can be achieved by E-SDM with resource (data rate and transmit power) adaptation. Accordingly, this scheme

\footnotetext{
Manuscript received March 7, 2007.

Manuscript revised May 28, 2007.

†The authors are with the Graduate School of Information Science and Technology, Hokkaido University, Sapporo-shi, 0600814 Japan.

a)E-mail: hn@ist.hokudai.ac.jp

b)E-mail: nishim@ist.hokudai.ac.jp

c) E-mail: ohgane@ist.hokudai.ac.jp

d)E-mail: ogawa@ist.hokudai.ac.jp

DOI: 10.1093/ietcom/e90-b.11.3197
}

can potentially provide enormous capacities and excellent communication quality in MIMO systems.

In a frequency-selective MIMO channel, orthogonal frequency division multiplexing (OFDM) transmission is robust to delay paths [8], [9], [17], [18]. The optimum processing at the transmitter in a MIMO-OFDM system is beamforming based on eigenvectors at each subcarrier to orthogonalize the MIMO channel [19], [20]. Also in MIMO single carrier transmission, we can apply eigenbeams to space-frequency domain equalization where the eigenbeam is calculated at each orthogonal frequency point [21]. However, since the computational complexity of SVD or eigenvalue decomposition (EVD) is very high, total calculation load of SVD or EVD increases in proportion to the number of active frequency points. Hence, it is necessary to reduce the load.

In addition, conventional SVD or EVD has another problem. This procedure includes phase ambiguity itself and is individually executed at each frequency point. Even if it would be possible to remove such phase ambiguity, the order of eigenvalues/eigenvectors may change at some frequency points in a frequency selective channel, and it is still difficult to find such order switching over discrete frequency points. Thus, frequency correlation (hereinafter we refer to it as frequency continuity) of the transmit weight is difficult to be maintained. These emphasize frequency selectivity of the effective channel, which is observed at the receiver through both effects of transmit weight and the MIMO channel. As a result, the impulse response has very large delay spread.

In a case without beamforming, it has been reported that the receiver can accurately estimate frequency-selective MIMO channels by using time windowing in both timeand frequency-domain estimation [22]-[27]. These schemes are commonly based on the concept of improving SNR by limiting the impulse response duration and suppressing the noise power outside the window. Unfortunately, it is difficult to employ these schemes to estimate the effective channel because of its large delay spread, as will be shown later. Maintaining frequency continuity of the effective channel is, therefore, one of the important issues in a beamforming system.

Choi and Heath proposed an interpolation-based beamforming scheme with considering phases of weight vectors in a MIMO-OFDM system with limited weight feedback [28]. They control the phases of eigenvectors to provide 
good interpolation property at the transmitter side. So, the method does not guarantee maintaining frequency continuity. Utilizing the method as a solution for frequency continuity is currently inappropriate.

To solve the issues raised above, in this paper we propose a pseudo eigenvector (PEV) scheme, which can reduce the calculation load and maintain frequency continuity of the effective channel. We also present the practical performance of the pseudo E-SDM (PE-SDM) transmission, in which PEVs are employed as transmit weights.

This paper is organized as follows. Section 2 defines the MIMO system model under a frequency-selective fading environment and introduces the E-SDM transmission. Section 3 describes the concept and procedure of PEV calculation and presents PEV properties. Section 4 proposes a time windowing scheme improving accuracy of effective channel estimation in the PE-SDM transmission. Section 5 reports the practical performance of the PE-SDM transmission applied to MIMO-OFDM systems. We draw conclusions from these results in Sect. 6.

\section{MIMO System Model and E-SDM}

We clarify a MIMO wireless system model under a frequency-selective fading environment. Note that in the following we discuss a baseband system. We assume that a MIMO system has $N_{\text {tx }}$ transmit (TX) antennas and $N_{\text {rx }}$ receive $(\mathrm{RX})$ antennas. To facilitate the following discussion in the frequency domain, it is assumed that a guard interval (GI) is added to the beginning of each transmitted symbol in multicarrier transmission such as OFDM or each transmitted block in single carrier one. This assumption ensures that the received sequence has periodicity within the FFT window duration and that there is no inter-symbol interference in the multicarrier case or inter-block interference in the single carrier case. When the transmitter beamforms at each frequency point to spatially multiplex $K$ substreams $\left(K \leq \min \left(N_{\mathrm{tx}}, N_{\mathrm{rx}}\right)\right)$, an $N_{\mathrm{rx}}$-dimensional $\mathrm{RX}$ signal vector at frequency point $f$ is expressed as

$$
\boldsymbol{r}(f)=\boldsymbol{H}_{F}(f) \boldsymbol{U}(f) \sqrt{\boldsymbol{P}(f)} \boldsymbol{s}(f)+\boldsymbol{n}(f),
$$

where $\boldsymbol{H}_{F}(f)$ is an $N_{\mathrm{rx}} \times N_{\mathrm{tx}}$ channel matrix, $\boldsymbol{U}(f)$ is an $N_{\text {tx }} \times K$ TX weight matrix, $\sqrt{\boldsymbol{P}(f)}$ is a $K$-dimensional diagonal matrix that is a square root of a $K$-dimensional TX power matrix $\boldsymbol{P}(f)=\operatorname{diag}\left[p_{1}(f), \ldots, p_{K}(f)\right], \boldsymbol{s}(f)$ is a $K$-dimensional TX signal vector, and $\boldsymbol{n}(f)$ is an $N_{\mathrm{rx}^{-}}$ dimensional thermal noise vector, which obeys a complex Gaussian process of zero mean and variance of $\sigma^{2}$. Here, we define an $N_{\mathrm{rx}} \times K$ effective channel matrix $\boldsymbol{B}_{F}(f)$ in the following equation

$$
\begin{aligned}
\boldsymbol{B}_{F}(f) & =\boldsymbol{H}_{F}(f) \boldsymbol{U}(f) \sqrt{\boldsymbol{P}(f)} \\
& =\left[\boldsymbol{b}_{F, 1}(f) \cdots \boldsymbol{b}_{F, K}(f)\right] .
\end{aligned}
$$

That is, the effective channel is composed of the actual channel and TX beams. Substituting (2) into (1) yields

$$
\boldsymbol{r}(f)=\boldsymbol{B}_{F}(f) \boldsymbol{s}(f)+\boldsymbol{n}(f) .
$$

Optimum beamforming is achieved by employing eigenvectors (hereinafter we refer to them as true eigenvectors (TEVs)) as TX weight vectors. Under an assumption of $K=\min \left(N_{\mathrm{tx}}, N_{\mathrm{rx}}\right)$, the SVD of the channel matrix is expressed as

$$
\boldsymbol{H}_{F}(f)=\boldsymbol{V}_{e}(f) \Sigma(f) \boldsymbol{U}_{e}^{H}(f),
$$

where

$$
\begin{aligned}
& \boldsymbol{V}_{e}(f)=\left[\boldsymbol{v}_{e, 1}(f) \cdots \boldsymbol{v}_{e, K}(f)\right] \quad\left(N_{\mathrm{rx}} \times K\right) \\
& \boldsymbol{U}_{e}(f)=\left[\boldsymbol{u}_{e, 1}(f) \cdots \boldsymbol{u}_{e, K}(f)\right] \quad\left(N_{\mathrm{tx}} \times K\right) \\
& \Sigma(f)=\operatorname{diag}\left[\sqrt{\lambda_{1}(f)}, \ldots, \sqrt{\lambda_{K}(f)}\right] \text {. }
\end{aligned}
$$

Here, $(\cdot)^{H}$ denotes the Hermitian transpose, both $\boldsymbol{v}_{e, i}(f)$ and $\boldsymbol{u}_{e, i}(f)(i=1, \ldots, K)$ are TEVs, and $\lambda_{1}(f), \ldots, \lambda_{K}(f)$ are eigenvalues in descending order. When we utilize $\boldsymbol{U}_{e}(f)(=$ $\left.\boldsymbol{H}_{F}^{H}(f) \boldsymbol{V}_{e}(f) \Sigma^{-1}(f)\right)$ as the TX weight matrix $\boldsymbol{U}(f)$, the receiver observes the effective channel as

$$
\boldsymbol{B}_{F, e}(f)=\boldsymbol{H}_{F}(f) \boldsymbol{U}_{e}(f) \sqrt{\boldsymbol{P}(f)} .
$$

In this case, the matrix $\boldsymbol{V}_{e}(f)$ enables the receiver to separate the spatially multiplexed signals without inter-substream interference as follows

$$
\begin{aligned}
\boldsymbol{y}(f) & =\boldsymbol{V}_{e}^{H}(f) \boldsymbol{r}(f) \\
& =\Sigma(f) \sqrt{\boldsymbol{P}(f)} \boldsymbol{s}(f)+\boldsymbol{V}_{e}^{H}(f) \boldsymbol{n}(f) .
\end{aligned}
$$

Equation (11) indicates that the SNR of the $i$ th detected substream is in proportion to $\lambda_{i}(f)$. This is the E-SDM scheme, which is the optimum transmission in a frequency-selective MIMO channel.

Obtaining TEVs at each frequency point, however, increases the computational load. Also, in general, TEVs are calculated without consideration of their frequency continuity so that they enlarge the delay spread of the impulse response of the effective channel, as will be demonstrated in Sect. 3.3. Addressing these issues, we propose the PEV scheme in the next section.

In the paper, we consider two multipath scenarios as illustrated in Fig. 1, where $T_{d}$ indicates the maximum time delay of paths. Scenario A is an environment where there are 16 sample-spaced i.i.d. Rayleigh paths $\left(T_{d}=15\right)$ with average $1-\mathrm{dB}$ decaying. Therefore, its frequency selectivity is relatively high. On the other hand, Scenario B has three

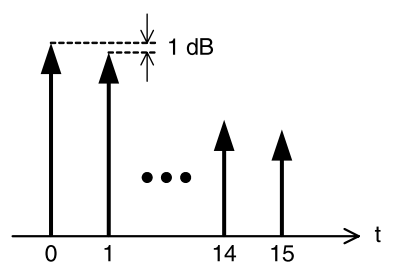

(a) Scenario A (16 paths, $\left.T_{d}=15\right)$

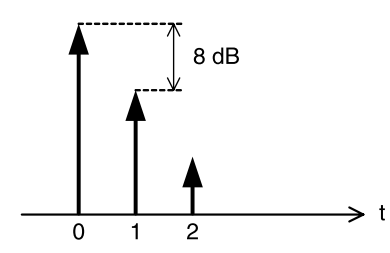

(b) Scenario B (3 paths, $\left.T_{d}=2\right)$
Fig. 1 Two multipath fading scenarios. 
sample-spaced i.i.d. Rayleigh paths $\left(T_{d}=2\right)$ with average 8$\mathrm{dB}$ decaying so that it results in lower frequency selectivity. In other words, Scenario B is close to a frequency-flat fading environment.

\section{Pseudo Eigenvector}

\subsection{Virtual RX Weight Matrix}

First, we define an $N_{\mathrm{rx}} \times N_{\mathrm{rx}} \mathrm{RX}$ autocorrelation function matrix $\boldsymbol{R}_{\mathrm{rx}}(\tau)$ by

$$
\begin{aligned}
\boldsymbol{R}_{\mathrm{rx}}(\tau) & =\frac{1}{\sqrt{N_{\mathrm{FFT}}}} \sum_{t=0}^{N_{\mathrm{FFT}}-1} \boldsymbol{H}_{T}(t) \boldsymbol{H}_{T}^{H}(t+\tau) \\
& =\frac{1}{\sqrt{N_{\mathrm{FFT}}}} \sum_{t=0}^{T_{d}} \boldsymbol{H}_{T}(t) \boldsymbol{H}_{T}^{H}(t+\tau),
\end{aligned}
$$

where both $t$ and $\tau$ denote time point indices, $N_{\mathrm{FFT}}$ stands for the FFT size, and $\boldsymbol{H}_{T}(t)$ represents the channel matrix of the path at time $t$, which can be obtained by applying the IFFT to $\boldsymbol{H}_{F}(f)$. The RX autocorrelation function matrix has the property of $\boldsymbol{R}_{\mathrm{rX}}(-\tau)=\boldsymbol{R}_{\mathrm{rx}}^{H}(\tau)$ and is also in a Fourier transform pair relationship with $\boldsymbol{H}_{F}(f) \boldsymbol{H}_{F}^{H}(f)$. That is, we have

$$
\boldsymbol{R}_{\mathrm{rx}}(\tau)=\mathcal{F}^{-1}\left[\boldsymbol{H}_{F}(f) \boldsymbol{H}_{F}^{H}(f)\right],
$$

where $\mathcal{F}^{-1}[\cdot]$ indicates the IFFT operation. In the function $\boldsymbol{R}_{\mathrm{rx}}(\tau)$, only $\boldsymbol{R}_{\mathrm{rx}}(0)$ is a non-negative Hermitian matrix. The sum of its diagonal elements $\operatorname{tr}\left[\boldsymbol{R}_{\mathrm{rX}}(0)\right]$ corresponds to the whole energy of the channel. Note that $\boldsymbol{R}_{\mathrm{rx}}(0)$ is equivalent to the sum or average of $\boldsymbol{H}_{F}(f) \boldsymbol{H}_{F}^{H}(f)$ over all the frequency points as follows

$$
\boldsymbol{R}_{\mathrm{rX}}(0)=\frac{1}{\sqrt{N_{\mathrm{FFT}}}} \sum_{f=0}^{N_{\mathrm{FFT}}-1} \boldsymbol{H}_{F}(f) \boldsymbol{H}_{F}^{H}(f) .
$$

We express the EVD of $\boldsymbol{R}_{\mathrm{rx}}(0)$ as

$$
\boldsymbol{R}_{\mathrm{rx}}(0)=\boldsymbol{V}_{0} \boldsymbol{\Lambda}_{0} \boldsymbol{V}_{0}^{H},
$$

where $\boldsymbol{\Lambda}_{0}$ is an $N_{\mathrm{rx}}$-dimensional diagonal matrix composed of descending ordered eigenvalues of $\boldsymbol{R}_{\mathrm{rx}}(0)$, and $\boldsymbol{V}_{0}$ is an $N_{\text {rx }}$-dimensional unitary matrix. Our proposal is based on using $\boldsymbol{V}_{0}^{H}$ as a tentative RX weight matrix. In the next subsection, we describe the method to obtain the TX weight matrix from $\boldsymbol{V}_{0}^{H}$.

\subsection{Pseudo Eigenvector Calculation}

In order to maintain the frequency continuity of the effective channel, the frequency continuity of the TX weight vectors must be accomplished. Here, we propose a PEV for the TX weight. The following describes the concept and the calculation procedure of the vectors.

We redefine $K=\min \left(N_{\mathrm{tx}}, N_{\mathrm{rx}}\right)$. Also, matrices $\boldsymbol{V}_{0, K}$ and $\Lambda_{0, K}$ are defined as an $N_{\mathrm{rx}} \times K$ matrix composed of the first $K$ column vectors of $V_{0}$ and a $K$-dimensional diagonal matrix composed of the first $K$ diagonal elements of $\boldsymbol{\Lambda}_{0}$, respectively. The matrix $\boldsymbol{V}_{0, K}^{H}$ can be considered as a frequency-flat $\mathrm{RX}$ weight matrix for the mean of $\boldsymbol{H}_{F}(f) \boldsymbol{H}_{F}^{H}(f)$ averaged over all frequencies. If the channel characteristic is frequency-flat, i.e., $\boldsymbol{H}_{F}(f)=\boldsymbol{H}_{F}, \boldsymbol{V}_{0, K}^{H}$ and $\boldsymbol{H}_{F}^{H} \boldsymbol{V}_{0, K} \sqrt{\Lambda_{0, K}}-1$ will be the optimum RX and TX weight matrices, respectively. Expanding this assumption, we can assume that $\boldsymbol{H}_{F}^{H}(f) \boldsymbol{V}_{0, K} \sqrt{\Lambda_{0, K}}-1$ approximates to the ideal TX matrix $\boldsymbol{U}_{e}(f)$ in the frequency-selective channel. It

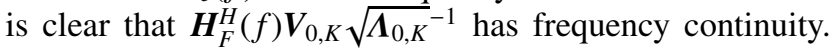
However, unlike $\boldsymbol{U}_{e}(f), \boldsymbol{H}_{F}^{H}(f) \boldsymbol{V}_{0, K}{\sqrt{\Lambda_{0, K}}}^{-1}$ is not composed of orthonormal vectors that are required for the TX weight vectors. Therefore, we ignore $\sqrt{\Lambda_{0, K}}-1$ and consider applying orthonormalization to column vectors of $\boldsymbol{H}_{F}^{H}(f) \boldsymbol{V}_{0, K}$ to obtain a PEV matrix. Here, we propose applying the GramSchmidt (GS) orthonormalization as

$$
\begin{aligned}
& \boldsymbol{U}_{e}^{\prime}(f)=\mathcal{G} \mathcal{S}\left[\boldsymbol{H}_{F}^{H}(f) \boldsymbol{V}_{0, K}\right] \\
& =\left[\begin{array}{lll}
\boldsymbol{u}_{e, 1}^{\prime}(f) & \cdots & \boldsymbol{u}_{e, K}^{\prime}(f)
\end{array}\right],
\end{aligned}
$$

where $\mathcal{G S}[\cdot]$ denotes the GS operation.

Tsunekawa et al. proposed a similar method, which obtains a TX weight matrix by GS processing in a MIMOOFDM system [29]. For simple hardware implementation, it orthonormalizes column vectors of the channel itself, i.e., $\mathcal{G S}\left[\boldsymbol{H}_{F}^{H}(f)\right]$, in descending order of their Euclidean norms. Orthonormalizing in this order is efficient in terms of obtaining vectors that are similar to TEVs. However, such ordering may cause frequency discontinuity of the obtained vectors because the order is not the same at all frequency points. (The effect and frequency discontinuity due to ordering will be discussed in Appendix.) Since our major purpose is to maintain frequency continuity of TX vectors, in (17) we naturally orthonormalize column vectors in the order of column number. The first column vector $\boldsymbol{u}_{e, 1}^{\prime}(f)$ has the best frequency continuity because only normalization is applied. Since subsequent column vectors are affected by both orthogonalization and normalization, their frequency continuity is expected to be less. However, since these processings are applied to the vectors originally having frequency continuity, subsequent column vectors also tend to have frequency continuity.

Consequently, the proposed method enables us to maintain frequency continuity of the vectors and the effective channel with a reduced calculation load. In the following, we show its frequency continuity and low numerical complexity in Sects. 3.3 and 3.4, respectively. It should be noted that the orthogonality of received substreams might be lost when the difference between PEVs and TEVs cannot be ignored. That is, $\boldsymbol{U}_{e}^{\prime} H_{(}(f) \boldsymbol{H}_{F}^{H}(f) \boldsymbol{H}_{F}(f) \boldsymbol{U}_{e}^{\prime}(f)$ is nearly, but not quite, a diagonal matrix. Therefore, since in spatial multiplexing there is inter-substream interference at the receiver, we need to suppress the interference to detect substreams. 


\subsection{Power Delay Profile of Effective Channels}

We demonstrate that PEVs can achieve frequency continuity of effective channels. Here, we define an impulse response matrix $\boldsymbol{B}_{T}(f)$ by

$$
\begin{aligned}
\boldsymbol{B}_{T}(t) & =\mathcal{F}^{-1}\left[\boldsymbol{B}_{F}(f)\right] \\
& =\left[\boldsymbol{b}_{T, 1}(t) \cdots \boldsymbol{b}_{T, K}(t)\right],
\end{aligned}
$$

where $\boldsymbol{b}_{T, i}(t)$ is an $N_{\mathrm{rx}}$-dimensional impulse response vector corresponding to the $i$ th substream. Although it is difficult to examine frequency continuity from the frequency-domain characteristics, we can easily see it in the time domain. That is, as the effective channel $\boldsymbol{B}_{F}(f)$ has better frequency continuity, its delay spread is lower. The delay spread for the $i$ th substream can be examined by calculating $E\left[\left\|\boldsymbol{b}_{T, i}(t)\right\|^{2}\right]$, where $E[\cdot]$ denotes an expectation, and $\|\cdot\|$ denotes the Euclidean norm. Accordingly, we evaluate frequency continuity of effective channels by using their delay spreads in this subsection.

Figure 2 illustrates mean power delay profiles of effective channels in a $4 \times 4$ MIMO system under the two multipath scenarios. In addition, their root mean square (RMS) delay spreads are listed in Table 1 . The FFT size was $N_{\mathrm{FFT}}=$ 64 , and TX weights with equal power were multiplied at all $N_{\text {FFT }}$ frequency points. We averaged over 100,000 different effective channels. It should be noted that, for TEV cases,

the EVD of $\boldsymbol{H}_{F}^{H}(f) \boldsymbol{H}_{F}(f)$ was calculated under a constraint forcing the first element of each TEV to be real.

Unlike actual channels $\boldsymbol{H}_{T}(t)$, effective channels have responses also in the region of $t<0$ as shown in Fig. 2 because TX beams have their own frequency characteristics, and their impulse responses are not causal. Since TEVs naturally do not have frequency continuity, they obviously cause large delay spread regardless of the multipath scenario and substream. This means that TEVs generally cause frequency discontinuity of the effective channels. Note that mean power delay profiles for TEV cases would show almost flat characteristics regardless of the scenario and sub-

Table 1 RMS delay spreads of effective channels for a $4 \times 4$ MIMO case.

\begin{tabular}{|l|c||c|c|}
\hline \multicolumn{2}{|c||}{} & \multicolumn{2}{c|}{ RMS delay spread [samples] } \\
\cline { 3 - 4 } \multicolumn{1}{|c|}{} & PEV & TEV \\
\hline \multirow{3}{*}{ Scenario A } & substream \#1 & 2.97 & 10.24 \\
& substream \#2 & 3.57 & 14.43 \\
& substream \#3 & 4.41 & 17.36 \\
& substream \#4 & 4.45 & 17.67 \\
\cline { 2 - 4 } & multipath & \multicolumn{2}{|c|}{3.47} \\
\hline \multirow{5}{*}{ Scenario B } & substream \#1 & 0.30 & 4.33 \\
& substream \#2 & 0.37 & 7.05 \\
& substream \#3 & 0.48 & 9.01 \\
& substream \#4 & 0.60 & 9.61 \\
\cline { 2 - 4 } & multipath & \multicolumn{2}{|c}{0.43} \\
\hline
\end{tabular}

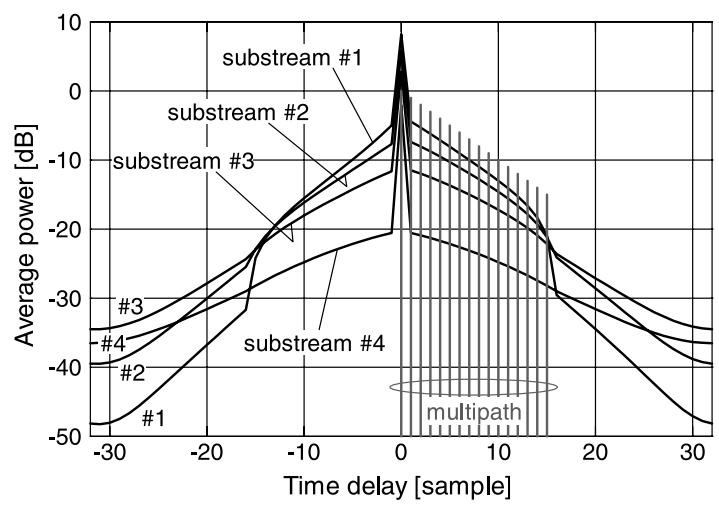

(a) Scenario A, PEV case

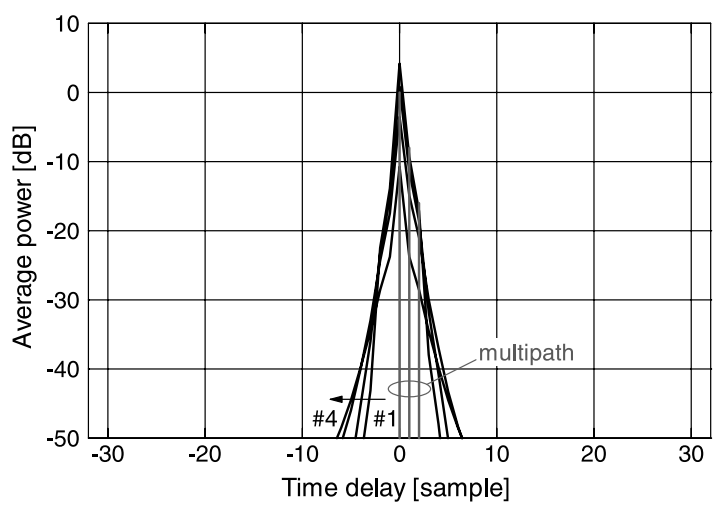

(c) Scenario B, PEV case

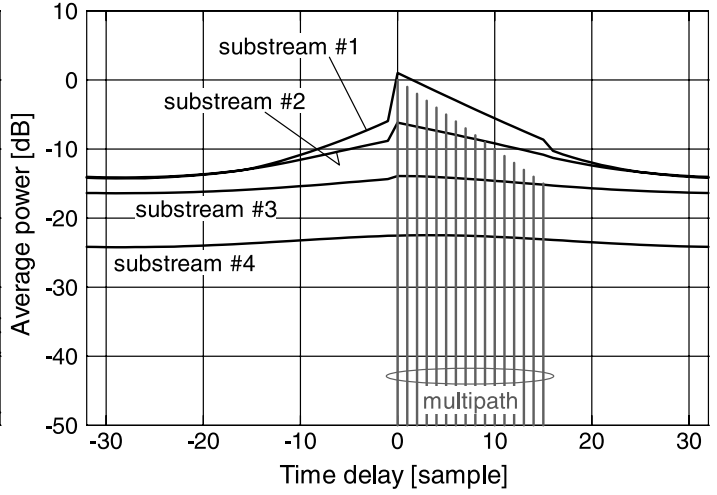

(b) Scenario A, TEV case

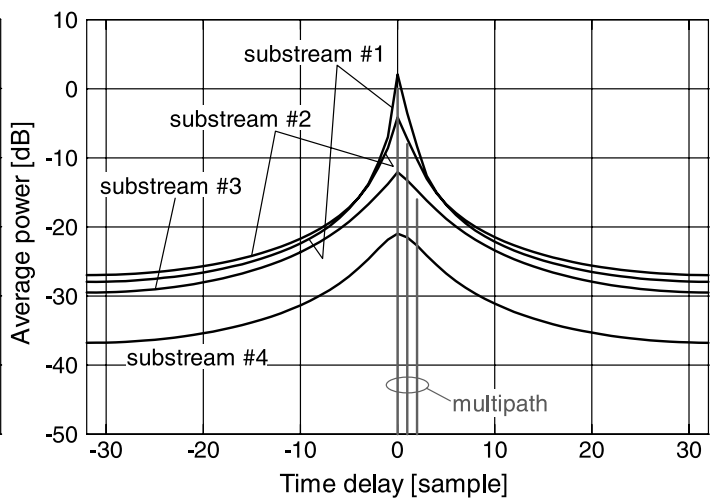

(d) Scenario B, TEV case

Fig. 2 Mean power delay profiles of effective channels in a $4 \times 4$ MIMO case. 
stream if TEVs were calculated without the constraint mentioned above. On the other hand, PEVs concentrate impulse responses in the region of $|t| \leq T_{d}$ and give high peaks at $t=0$ regardless of the substream. Also, the responses in the region of $|t|>T_{d}$ are at a significantly low level, especially for Scenario B. This means that the delay spreads of effective channels are highly reduced by using PEVs. Consequently, we can say that PEVs as TX weights can maintain frequency continuity of effective channels. Thus, time windowing can be utilized to improve accuracy of the effective channel estimation, as will be shown later.

\subsection{Numerical Complexity}

The proposed PEV scheme has lower computational complexity compared with calculation of TEVs. To verify it, we examined their mean and maximum numbers of complex multiplications per frame for $2 \times 2$ and $4 \times 4$ MIMO cases with the same setup as the previous subsection, as listed in Table 2. The complexity in the PEV scheme was examined for the EVD of $\boldsymbol{R}_{\mathrm{rx}}(0)$ and the GS operation of $\boldsymbol{H}_{F}^{H}(f) \boldsymbol{V}_{0, K}$, and that in the TEV scheme was examined for the EVD of $\boldsymbol{H}_{F}^{H}(f) \boldsymbol{H}_{F}(f)$ for comparison, where EVD was solved by Jacobi method. Therefore, it should be noted that they do not contain calculation of FFT, matrix multiplication, and the summation in (13). For the TEV scheme, to speed up the convergence in EVD, $\boldsymbol{U}_{e}(f-1)$ obtained at the adjacent frequency point $f-1$ was utilized as an initial unitary matrix for $\boldsymbol{U}_{e}(f)$. Since Scenario B has lower frequency selectivity, this initial value setting decreases number of iterations in EVD. Although, for the $2 \times 2$ MIMO case, both schemes have almost the same complexity, for the $4 \times 4$ MIMO case the PEV scheme can reduce it to about 1/11 (Scenario A) -1/9 (Scenario B) of that in the TEV one when comparing mean numbers of complex multiplications. Even in comparison of maximum numbers which are important factors for evaluating implementability of algorithms, PEVs for the $4 \times 4$ MIMO can be computed with about $1 / 8$ complexity of TEV calculation in both scenarios. Independence of complexity on the multipath scenario for the $2 \times 2$ MIMO case is because both the GS orthonormalization and a two-dimensional eigenvalue problem can be solved with simple equations of fixed complexity (without iterative calculations).

Table 2 Mean and maximum numbers of complex multiplications per fading state for $2 \times 2$ and $4 \times 4$ MIMO cases.

\begin{tabular}{|c|c||c|c|}
\hline \multicolumn{2}{|c||}{} & \multicolumn{2}{c|}{$\begin{array}{c}\text { Number of complex multiplications } \\
\text { (mean/max) }\end{array}$} \\
\cline { 3 - 4 } \multicolumn{2}{|c|}{} & PEV & TEV \\
\hline \hline \multirow{2}{*}{$2 \times 2$} & Scenario A & $1,074 / 1,074$ & $1,152 / 1,152$ \\
& Scenario B & $1,074 / 1,074$ & $1,152 / 1,152$ \\
\hline \multirow{2}{*}{$4 \times 4$} & Scenario A & $6,581 / 11,784$ & $73,674 / 86,604$ \\
& Scenario B & $6,453 / 11,784$ & $59,994 / 88,338$ \\
\hline
\end{tabular}

\subsection{Pseudo Eigenvalue}

Here, let us define a pseudo eigenvalue $\lambda_{i}^{\prime}(f)$ of the $i$ th substream at frequency point $f$ by

$$
\begin{aligned}
\lambda_{i}^{\prime}(f) & =\left\|\boldsymbol{H}_{F}(f) \boldsymbol{u}_{e, i}^{\prime}(f)\right\|^{2} \\
& =\boldsymbol{u}_{e, i}^{\prime H}(f) \boldsymbol{H}_{F}^{H}(f) \boldsymbol{H}_{F}(f) \boldsymbol{u}_{e, i}^{\prime}(f) .
\end{aligned}
$$

The pseudo eigenvalue $\lambda_{i}^{\prime}(f)$ is in proportion to the received SNR of the $i$ th substream in the case of single-beam transmission using $\boldsymbol{u}_{e, i}^{\prime}(f)$. In the above equation, $\lambda_{i}^{\prime}(f)$ becomes the true eigenvalue $\lambda_{i}(f)$ by replacing $\boldsymbol{u}_{e, i}^{\prime}(f)$ by the $i$ th TEV $\boldsymbol{u}_{e, i}(f)$.

Figure 3 shows examples of true and pseudo eigenvalues for a $4 \times 4$ MIMO system under the two multipath scenarios. Since both true and pseudo eigenvalues for Scenario A fluctuate in the frequency domain, we can say that the scenario has high frequency selectivity. On the other hand, for Scenario B its fading appears closer to frequencyflat fading, which is the assumed condition in the PEV calculation as described in Sect. 3.2. Thus, pseudo eigenvalues for Scenario B are in good agreement with eigenvalues so that PEVs are expected to be similar to TEVs. However, especially for Scenario A, some pseudo eigenvalues do not agree well with corresponding true eigenvalues. It is conjectured that PEVs in such regions mismatch with the channel, and that inter-substream interference may be larger. Note

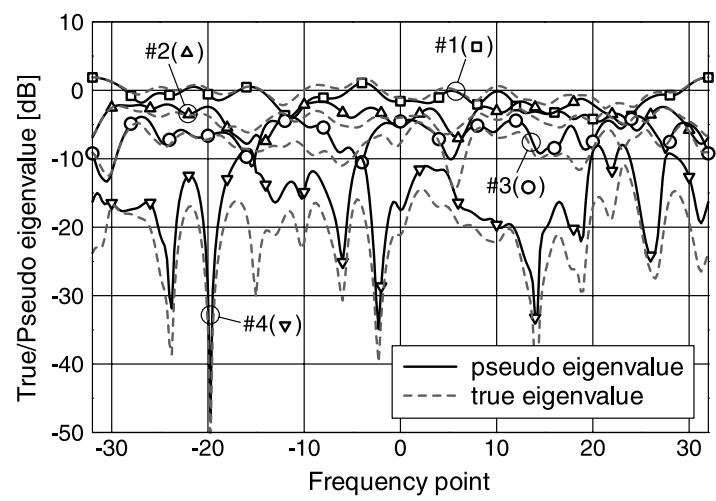

(a) Scenario A

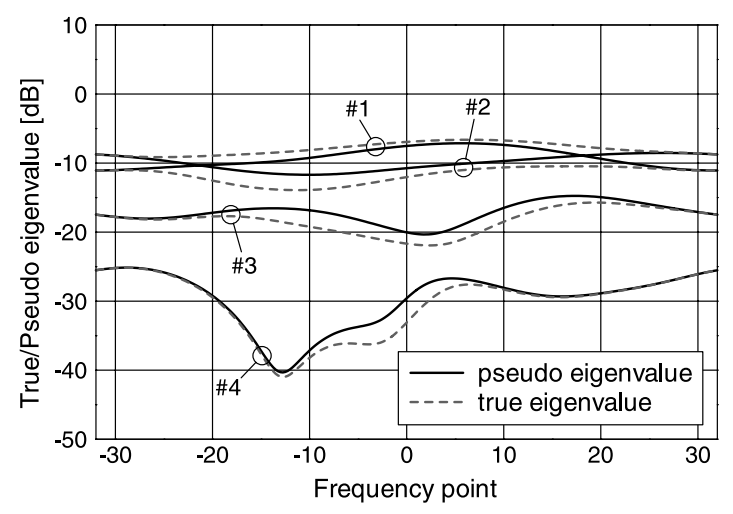

(b) Scenario B

Fig. 3 Examples of pseudo and true eigenvalues for a $4 \times 4$ MIMO case. 
that, especially for Scenario A, while true eigenvalues are in descending order regardless of the frequency point, the order of pseudo eigenvalues can change depending on the frequency selectivity. This is expected to relate to high frequency continuity, i.e., low delay spreads, of effective channels shown in Fig. 2.

\section{Estimation Scheme of Effective Channel in PE-SDM}

We clarified characteristics of the PEVs in Sect. 3. In this section, we propose an effective channel estimation scheme in PE-SDM transmission, in which PEVs are employed as TX weight vectors. Although a beamforming technique in the frequency domain can be applied to single carrier systems [21], in this paper we apply it to MIMO-OFDM.

\subsection{Frequency Domain Estimation of Effective Channel}

We presume that the data frame has a long preamble to estimate the effective channel at the receiver. The preamble is composed of $N_{\mathrm{LP}}=2^{n}$ OFDM symbols, which have a space-time orthogonal Walsh-Hadamard code, and is different in each substream. The number of the training symbols is determined by the maximum number of substreams $K_{\max }=\min \left(N_{\mathrm{tx}}, N_{\mathrm{rx}}\right)$. For example, since $K_{\max }=4$ in a $4 \times 4$ MIMO system, the preamble has $N_{\mathrm{LP}}=2^{2}=4$ training symbols to separate $K_{\max }$ substreams at the receiver as shown in Fig. 4. The receiver can estimate the effective channel of the desired substream in the frequency domain by linearly combining the $N_{\mathrm{LP}}$ symbols after the correspondent code multiplication [20].

\subsection{Proposed Time-Windowing Scheme in PE-SDM}

In addition to the above channel estimation method, we can improve the accuracy of the estimated effective channel in PE-SDM by applying the following simple processing. As stated in Sect. 3.3, the impulse responses of effective channels in PE-SDM are concentrated in the range of $|t| \leq T_{d}$ and are at a significantly lower level in $|t|>T_{d}$ (Fig. 2). Accordingly, when the effective channel is estimated using the above method, its impulse response in $|t|>T_{d}$ ought to be dominated by thermal noise. Then we propose to reduce thermal noise components included in the effective channel estimates by time windowing. For the sake of simplicity, in this paper we consider applying a simple time window function as illustrated in Fig. 5, where $T_{w}$ is defined as the time window width, and $G_{\text {out }}$ denotes the attenuation level outside the window $\left(G_{\text {out }} \leq 1\right)$. The impulse response, which is obtained by applying the IFFT to the effective channel estimate, is multiplied by the window. When we set $T_{w} \geq T_{d}$, we can efficiently capture the impulse response concentrated in $|t| \leq T_{d}$, and thermal noise components outside the window, i.e., $|t|>T_{w}$, are reduced. However, when some subcarriers are not used for transmission, such as guard bands, the IFFT distorts the impulse response [24]. To avoid this, values at the unused subcarriers are interpolated before the IFFT. Under the assumption that the effective channel is cyclic in the frequency domain, linear interpolation is sim-

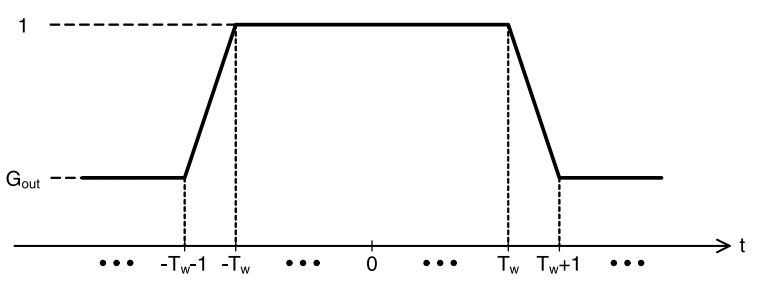

Fig. 5 Time window function

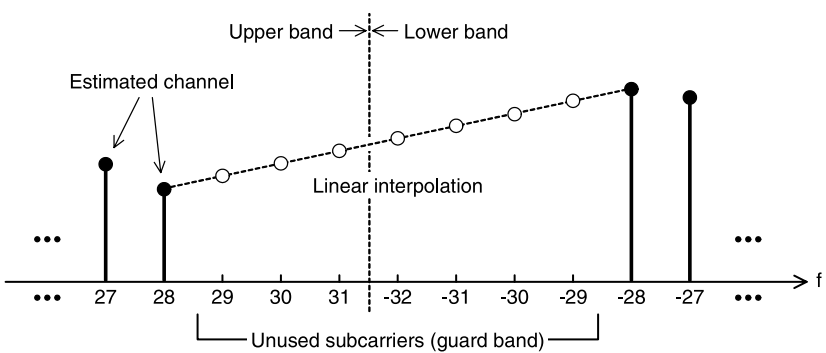

Fig. 6 Concept of linear interpolation of an estimated channel in the frequency domain $\left(N_{\mathrm{FFT}}=64\right)$.

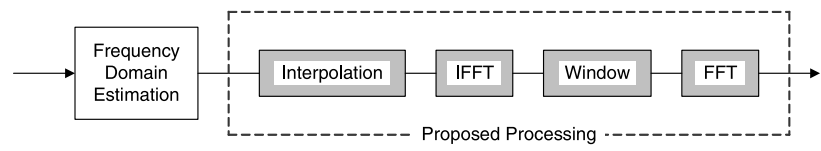

Fig. 7 Process flow chart of effective channel estimation with time windowing.

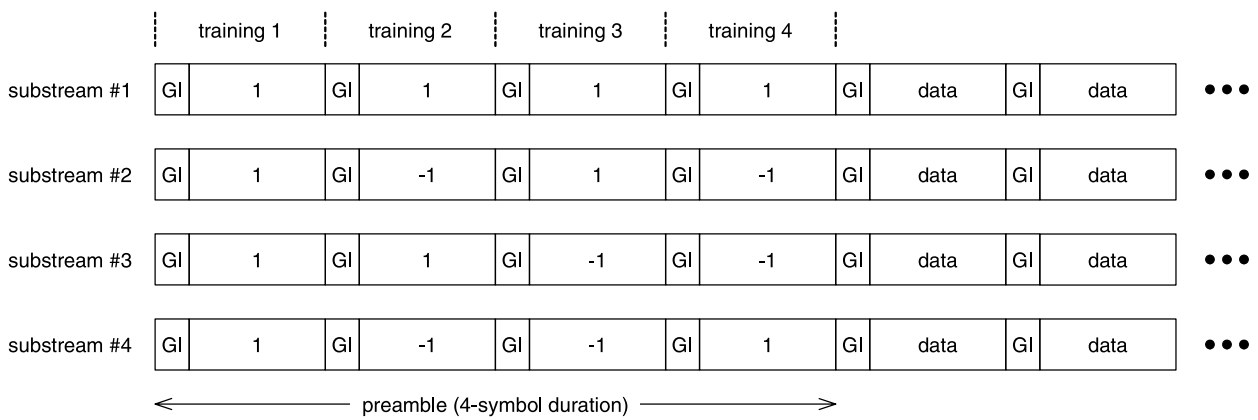

Fig. 4 Frame format in a case of $N_{\mathrm{LP}}=4$. 
ply done by using estimated values of the used subcarriers, which are the closest to unused subcarriers (Fig. 6). After time windowing, we obtain the refined effective channel estimate by applying the FFT to the impulse response. A process flow chart of effective channel estimation with time windowing is illustrated in Fig. 7.

\section{Computer Simulations}

To evaluate the proposed pseudo eigenbeam technique and time windowing scheme, we examined bit error rate (BER) performance of PE-SDM comparing with that of E-SDM by computer simulations.

\subsection{System Structure}

Figure 8 illustrates transmitter and receiver structures. A transmitted data sequence is encoded and randomly interleaved before multiplexing. This process yields an effect of space-frequency diversity in a decoded sequence at the receiver. Then encoded bits are mapped to constellations for each substream and beamformed based on power-allocated weight matrices $\boldsymbol{U}(f) \sqrt{\boldsymbol{P}(f)}$ at all subcarriers. According to the IEEE802.11a standard [7], a modulation scheme is common to all the subcarriers in each substream. We also assume that TX power allocation is common to all the subcarriers, i.e., $\boldsymbol{P}(f)=\boldsymbol{P}$, to simplify TX processing and not to enlarge the delay spread of the effective channel.

At the receiver, GIs are removed from the received signals, and then the FFT is applied to them. Substreams are detected at each subcarrier by a spatial filter based on the effective channel estimate. The demultiplexed sequence are deinterleaved and decoded, and then we obtain an estimated data sequence.

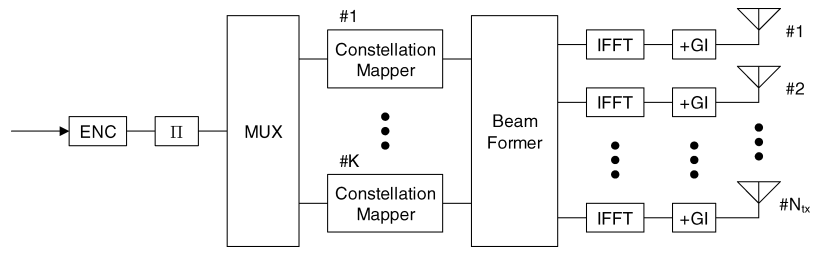

(a) Transmitter structure

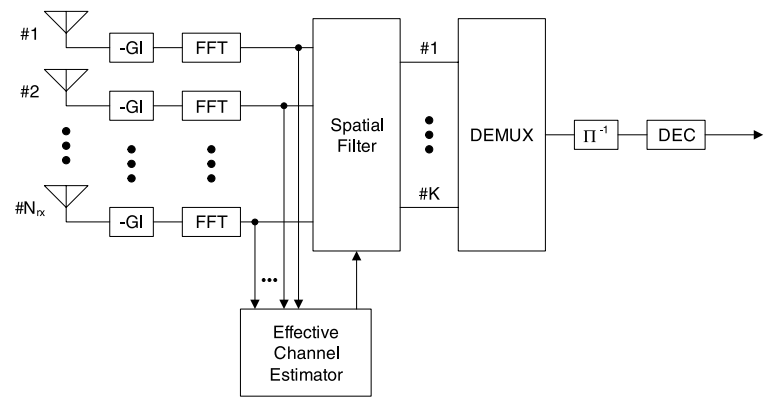

(b) Receiver structure

Fig. 8 Block diagram.

\subsection{Simulation Conditions}

Table 3 lists MIMO-OFDM simulation parameters. We examined the performance for $2 \times 2$ and $4 \times 4$ MIMO systems. Referring to the IEEE802.11a standard and contents in the IEEE802.11n standardization [7], the FFT size was set to $N_{\mathrm{FFT}}=64$, and 56 subcarriers were used for transmission, except for those in guard bands and at the center as illustrated in Fig. 9. Each OFDM symbol had 80 samples because of the GI duration of 16 samples.

It was assumed that the transmitter had perfect CSI by feedback through an error-free channel in a frequency division duplex system [20], [28], [30] or by channel estimation at the transmitter in a time division duplex system [15], [31]. On the other hand, it was assumed that only the number of substreams and modulation schemes were known at the receiver except the "perfect CSI case" where the CSI is also known. In the estimated CSI case, the time window width $T_{w}$ was set to $T_{d}$ under the assumption that the receiver could accurately estimate the maximum time delay of paths. The attenuation level $G_{\text {out }}=-10 \mathrm{~dB}$ was used because it provided stable improvement of effective channel estimates in various MIMO cases. The minimum mean square error (MMSE) criterion was used for determining spatial filter weights. A $K \times N_{\text {rx }}$ MMSE weight matrix $\boldsymbol{W}(f)$ at subcarrier $f$ is given by

$$
\begin{aligned}
\boldsymbol{W}(f) & =\hat{\boldsymbol{B}}_{F}^{H}(f)\left\{\hat{\boldsymbol{B}}_{F}(f) \hat{\boldsymbol{B}}_{F}^{H}(f)+\sigma^{2} \boldsymbol{I}_{N_{\mathrm{rx}}}\right\}^{-1} \\
& =\left[\boldsymbol{w}_{1}(f) \cdots \boldsymbol{w}_{K}(f)\right]^{T},
\end{aligned}
$$

Table 3 Simulation parameters.

\begin{tabular}{c|c}
\hline \hline MIMO systems & $2 \times 2\left(N_{\mathrm{LP}}=2\right), 4 \times 4\left(N_{\mathrm{LP}}=4\right)$ \\
\hline No. of FFT points & $N_{\mathrm{FFT}}=64$ \\
\hline No. of subcarriers & 56 \\
\hline GI duration & 16 samples \\
\hline Channel model & $\begin{array}{c}\text { Quasi-static i.i.d. Rayleigh fading } \\
\text { Scenario A, Scenario B }\end{array}$ \\
\hline Substream detection & MMSE spatial filtering \\
\hline Encoding & Convolutional code \\
\hline Decoding & Soft-decision Viterbi algorithm \\
\hline No. of data symbols & 8 \\
\hline Bit rate & 8 coded bits/symbol/subcarrier \\
\hline Modulation schemes & QPSK, 16QAM, 64QAM, 256QAM \\
\hline Substream quality & E-SDM: $E\left[\lambda_{i}(f)\right] / \sigma^{2}$ \\
\hline No. of trial frames & PE-SDM: $E\left[\lambda_{i}^{\prime}(f)\right] / \sigma^{2}$ \\
\hline \hline
\end{tabular}

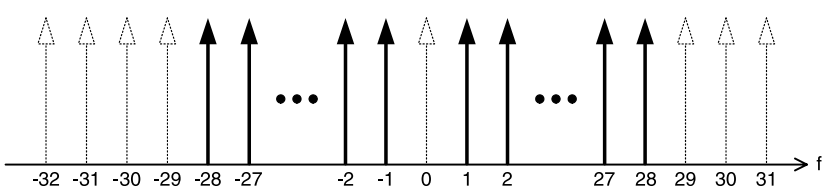

Fig. 9 Subcarrier arrangement. 
where $\hat{\boldsymbol{B}}_{F}(f)$ indicates an effective channel estimate, $\boldsymbol{I}_{N_{\mathrm{Ix}}}$ denotes an $N_{\mathrm{rx}}$-dimensional unit matrix, and $(\cdot)^{T}$ means the transpose. A $K$-dimensional MMSE output vector is obtained by multiplying $\boldsymbol{r}(f)$ by $\boldsymbol{W}(f)$. A data sequence was coded by a convolutional encoder with constraint length of three and coding rate of $1 / 2$. A data frame was composed of eight OFDM symbols. The random-interleaver size corresponded to the frame size. Soft-decision Viterbi decoding was applied. A soft-input decoder requires the likelihood of each bit. For the sake of simplicity, in each case of corresponding bit 1 or 0, we used the squared Euclidean distance between the output symbol and the closest replica symbol candidate as its log-likelihood [32]. In order to reflect channel quality depending on the substream and subcarrier, we multiplied the obtained log-likelihood by the corresponding SINR of the MMSE spatial filter output, i.e., $\boldsymbol{w}_{i}^{T}(f) \hat{\boldsymbol{b}}_{F, i}(f) /\left\{1-\boldsymbol{w}_{i}^{T}(f) \hat{\boldsymbol{b}}_{F, i}(f)\right\}[33]$.

Eight coded bits were assigned per OFDM symbol per subcarrier. We prepared four modulation schemes: QPSK, 16 QAM, 64 QAM, and 256 QAM. Under these conditions and total TX power constraint, the transmitter optimally determines modulation scheme and TX power per substream so as to give the minimum BER averaged over utilized substreams after Viterbi decoding [13], [34]. We employed mean eigenvalue $E\left[\lambda_{i}(f)\right]$ or mean pseudo eigenvalue $E\left[\lambda_{i}^{\prime}(f)\right]$ averaged over all subcarriers in each substream as the substream-quality measure in the decision.

\subsection{Simulation Results}

Figure 10 shows the average BER performance versus normalized total TX power for the case of perfect CSI at the receiver. Here, the normalized total TX power, which is introduced for fair comparison of PE-SDM and E-SDM, denotes the total TX power normalized to the TX power yielding average $E_{s} / N_{0}$ of $0 \mathrm{~dB}$ in a case of single-antenna OFDM transmission in the corresponding multipath fading scenario. Thus, note that actual TX power depends on the total power of multipaths.

It is evident that the performance for the $4 \times 4 \mathrm{MIMO}$ system is significantly improved compared with that for the $2 \times 2$ MIMO system regardless of PE-SDM/E-SDM and the scenario. This is yielded by an increase in the MIMO channel capacity. Let us compare the basic performance of PESDM with that of E-SDM. In E-SDM, since the transmitter utilizes TEVs of the channel at each subcarrier, the optimum beamforming gives excellent performance in both $2 \times 2$ and $4 \times 4$ MIMO systems. Compared with E-SDM, the performance for PE-SDM deteriorates due to gain loss, which occurs in reducing inter-substream interference by spatial filtering. At the BER of $10^{-5}$, the degradation of PE-SDM for Scenario $\mathrm{A}$ is about $2 \mathrm{~dB}$ and $1 \mathrm{~dB}$ for $2 \times 2$ and $4 \times 4 \mathrm{MIMO}$ cases, respectively. However, that for Scenario B is less than $0.5 \mathrm{~dB}$ for both MIMO cases. The difference between the scenarios is due to accuracy of produced PEVs as stated in Sect. 3.5. The PE-SDM scheme is, hence, more effective in lower delay spread environments.

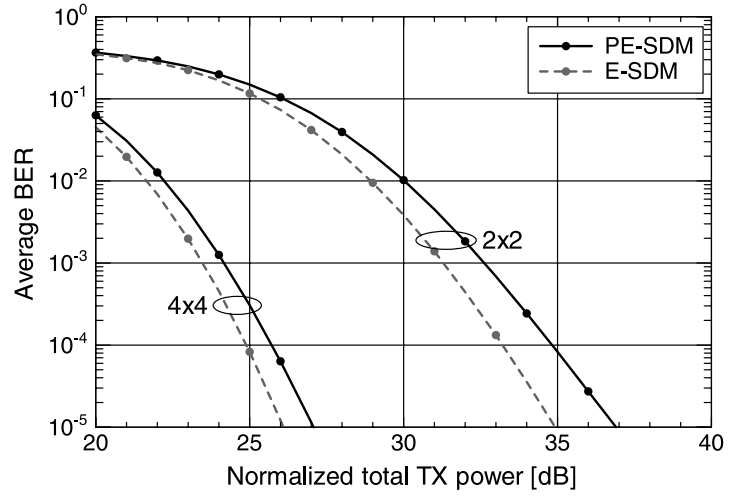

(a) Scenario A

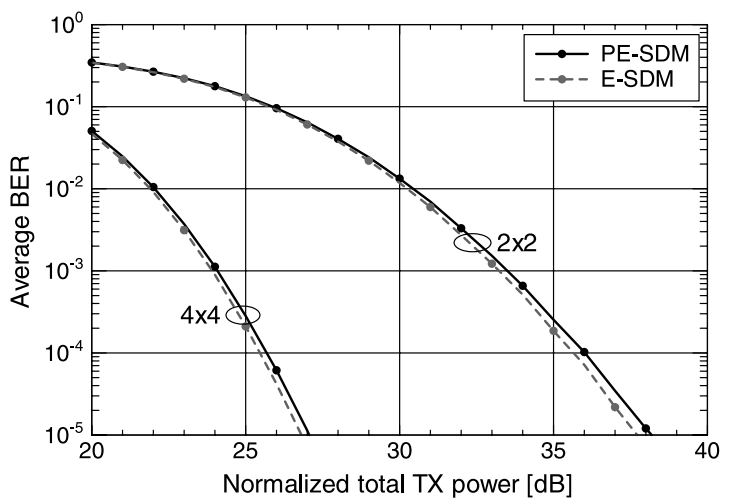

(b) Scenario B

Fig. 10 Average BER performance for the perfect CSI case.

The BER performance for the estimated CSI case is shown in Fig. 11. When the receiver estimates the effective channel only in the frequency domain, both the E-SDM and PE-SDM performances deteriorate by $2-3 \mathrm{~dB}$ compared with the perfect CSI case regardless of the number of antennas. However, with time windowing in addition to the frequency-domain estimation, the PE-SDM obtains about $1 \mathrm{~dB}$ and $2 \mathrm{~dB}$ gain for Scenario A and Scenario B, respectively. This implies that the impulse responses of the effective channels in the PE-SDM are well concentrated within the window and that thermal noise components outside the window are effectively reduced. Because of these benefits, the PE-SDM performance is almost the same as the E-SDM one for Scenario A, and the PE-SDM for Scenario B provides better performance than the E-SDM. The significant gain for Scenario B is because the receiver could narrow the time window width optimally since we assumed that it could accurately estimate the maximum time delay $T_{d}$. It is difficult in practice, however, to achieve such accurate estimation, and the time window width $T_{w}$ depends on the delay profile of the effective channel, i.e., its frequency continuity. In a practical case where $T_{w}$ is set more roughly, the effect of the time windowing may decrease. Therefore, setting of $T_{w}$ and window shape should be examined in our future work. Note that error floors are observed for the E-SDM performance with time windowing regardless of the fading scenario and number of antennas because time windowing 


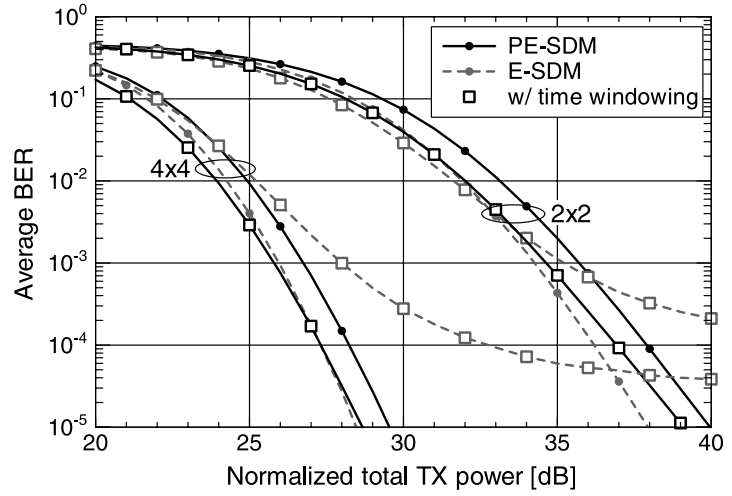

(a) Scenario A

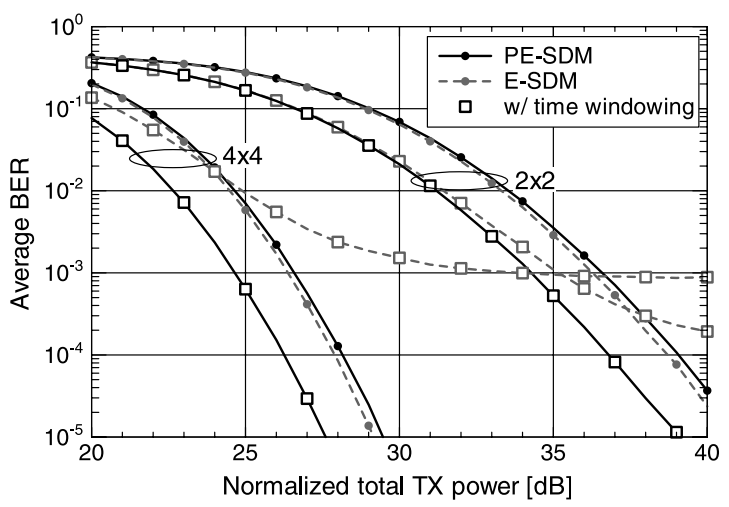

(b) Scenario B

Fig. 11 Average BER performance for the estimated CSI case.

Table 4 Maximum numbers of complex multiplications at the receiver per frame for conventional frequency-domain channel estimation (FDCE), MMSE weight calculation, and time windowing (TW).

\begin{tabular}{|c||c|c|}
\hline \multicolumn{1}{|c||}{} & \multicolumn{2}{c|}{$\begin{array}{c}\text { Number of complex multiplications } \\
\text { (maximum value) }\end{array}$} \\
\cline { 2 - 3 } & FDCE + MMSE & TW \\
\hline \hline $\begin{array}{c}2 \times 2 \text { MIMO } \\
(2 \text { substreams })\end{array}$ & $1,376+1,876=3,252$ & 1,566 \\
\hline $\begin{array}{c}4 \times 4 \text { MIMO } \\
(4 \text { substreams })\end{array}$ & $6,528+10,920=17,448$ & 6,264 \\
\hline
\end{tabular}

caused distortion in the impulse responses of the effective channel estimates as evidenced by Figs. 2(b), 2(d), and 5.

Here, let us discuss additional complexity at the receiver for time windowing, separately from the numerical complexity required at the transmitter for obtaining PEVs/TEVs (see Sect.3.4). We compare the complexity with substantial calculation at the receiver composed of conventional frequency-domain channel estimation (FDCE) and MMSE weight generation. Table 4 lists maximum values of numbers of complex multiplications needed at the receiver for FDCE, MMSE, and time windowing. These numbers were counted under a full-substream transmission case in each MIMO system, i.e., two-substream transmission in $2 \times 2$ MIMO and four-substream transmission in $4 \times 4 \mathrm{MIMO}$. We can see that time windowing costs $48.2 \%$ and $35.9 \%$ increases in computational complexity in $2 \times 2$ and $4 \times 4$ MIMO cases, respectively, from the substantial calculation load (FDCE + MMSE). As illustrated in Fig. 7, time window processing is composed of interpolation, IFFT, windowing, and FFT, and about $98 \%$ cost for time windowing is occupied by FFT and IFFT. Thus, the hardware implementation cost would be negligible if we can reuse the FFT and IFFT circuits for other purposes.

\section{Conclusions}

We have proposed pseudo eigenvectors for frequencyselective MIMO channels. They can be calculated with less complexity than that of conventional EVD or SVD, and they can maintain frequency continuity of the effective channel observed at the receiver. Although the subchannel orthogonality in PE-SDM is lost due to the disagreement between the pseudo and true eigenvectors, we confirmed less performance degradation in a less frequency-selective fading environment, and it is possible to compensate for the degradation by the channel estimation improvement arising from the frequency-continuity property. Thus, when the receiver incorporates a channel estimator exploiting the property such as time windowing, PE-SDM can provide almost the same or even better performance compared with E-SDM.

As stated in Sect. 3.2, the calculation of pseudo eigenvectors gives the priority of frequency continuity to the first substream. Our future work will involve accurately maintaining the frequency continuity of substreams that are subsequent to the first one. Also, optimization of time windowing and improvement of the TX resource control algorithm in frequency-selective fading should be examined.

\section{Acknowledgement}

This work was supported in part by a Research Fellowship for Young Scientists from the Japan Society for the Promotion of Science.

\section{References}

[1] G.J. Foschini and M.J. Gans, "On limits of wireless communications in a fading environment when using multiple antennas," Wirel. Pers. Commun., vol.6, pp.311-335, March 1998.

[2] G.D. Golden, G.J. Foschini, R.A. Valenzuela, and P.W. Wolniansky, "Detection algorithm and initial laboratory results using VBLAST space-time communication architecture," IEE Electron. Lett., vol.35, no.1, pp.14-16, Jan. 1999.

[3] I.E. Telatar, "Capacity of multi-antenna Gaussian channels," Euro. Trans. Telecommun., vol.1, no.6, pp.585-595, Nov./Dec. 1999.

[4] D. Gesbert, M. Shafi, D.S. Shiu, P. Smith, and A. Naguib, "From theory to practice: An overview of MIMO space-time coded wireless systems," IEEE J. Sel. Areas Commun., vol.21, no.2, pp.281-302, April 2003.

[5] A.J. Paulraj, D.A. Gore, R.U. Nabar, and H. Bölcskei, "An overview of MIMO communications - A key to gigabit wireless," Proc. IEEE, vol.92, no.2, pp.198-218, Feb. 2004.

[6] E. Biglieri, R. Calderbank, A. Constantinides, A. Goldsmith, A. Paulraj, and H.V. Poor, MIMO Wireless Communications, Cambridge University Press, New York, 2007. 
[7] IEEE802.11, The Working Group Setting the Standards for Wireless LANs, http://www.ieee802.org/11/

[8] S. Nanda, R. Walton, J. Ketchum, M. Wallace, and S. Howard, "A high-performance MIMO OFDM wireless LAN," IEEE Commun. Mag., vol.43, no.2, pp.101-109, Feb. 2005.

[9] A. Ghosh, D.R. Wolter, J.G. Andrews, and R. Chen, "Broadband wireless access with WiMax/802.16: Current performance benchmarks and future potential," IEEE Commun. Mag., vol.43, no.2, pp.129-136, Feb. 2005.

[10] K. Ban, M. Katayama, T. Yamazato, and A. Ogawa, "Joint optimization of transmitter/receiver with multiple transmit/receive antennas in band-limited channels," IEICE Trans. Commun., vol.E83-B, no.8, pp.1697-1704, Aug. 2000.

[11] H. Sampath, P. Stoica, and A. Paulraj, "Generalized linear precoder and decoder design for MIMO channels using the weighted MMSE criterion,” IEEE Trans. Commun., vol.49, no.12, pp.2198-2206, Dec. 2001.

[12] A. Scaglione, P. Stoica, S. Barbossa, G.B. Giannakis, and H. Sampath, "Optimal designs for space-time linear precoders and decoders," IEEE Trans. Signal Process., vol.50, no.5, pp.1051-1064, May 2002.

[13] K. Miyashita, T. Nishimura, T. Ohgane, Y. Ogawa, Y. Takatori, and K. Cho, "High data-rate transmission with eigenbeam-space division multiplexing (E-SDM) in a MIMO channel," Proc. IEEE VTC2002Fall, vol.3, pp.1302-1306, Sept. 2002.

[14] S. Ting, K. Sakaguchi, and K. Araki, "Performance analysis of MIMO eigenmode transmission system under realistic channel and system conditions," IEICE Trans. Commun., vol.E87-B, no.8, pp.2222-2232, Aug. 2004

[15] G. Lebrun, J. Gao, and M. Faulkner, "MIMO transmission over a time-varying channel using SVD," IEEE Trans. Wireless Commun. vol.4, no.2, pp.757-764, March 2005.

[16] L. Dai, S-D. Zhou, H-R. Zhuang, and Y. Yao, "Closed-loop MIMO architecture based on water-filling," IEE Electon. Lett., vol.38, no.25, pp.1718-1720, Dec. 2002.

[17] H. Sampath, S. Talwar, J. Tellado, V. Erceg, and A. Paularj, "A fourth-generation MIMO-OFDM broadband wireless system: Design, performance, and field trial results," IEEE Commun. Mag., vol.40, no.9, pp.143-149, Sept. 2002.

[18] G.G. Raleigh and V.K. Jones, "Multivariate modulation and coding for wireless communication," IEEE J. Sel. Areas Commun., vol.17, no.5, pp.851-866, May 1999.

[19] L.H. Doan, S.H. Ting, K. Sakaguchi, and K. Araki, "Hierarchical subgroup power and modulation coding adaptation - A new frequency-space link adaptation scheme in MIMO-OFDM eigenmode adaptive transmission system," Proc. IEEE VTC2005-Spring, vol.2, pp.668-672, May/June 2005.

[20] Y. Ohwatari, B.H. Phu, Y. Ogawa, T. Nishimura, and T. Ohgane, "Reduction of the amount of channel state information feedback in MIMO-OFDM eigenbeam-space division multiplexing systems," Proc. IEEE VTC2006-Fall, in CD-ROM, Sept. 2006.

[21] K. Ozaki, A. Nakajima, and F. Adachi, "Frequency-domain eigenbeam-SDM and equalization for high speed data transmissions," Proc. IEEE VTC2006-Fall, in CD-ROM, Sept. 2006.

[22] Y. Li, N. Seshadri, and S. Ariyavisitakul, "Channel estimation for OFDM system with transmitter diversity in mobile wireless channels," IEEE J. Sel. Areas Commun., vol.17, no.3, pp.461-471, March 1999

[23] Y. Li, "Simplified channel estimation for OFDM systems with multiple transmit antennas," IEEE Trans. Wireless Commun., vol.1, no.1, pp.67-75, Jan. 2002.

[24] Y. Ogawa, K. Nishio, T. Nishimura, and T. Ohgane, "Channel estimation and signal detection for space division multiplexing in a MIMO-OFDM system," IEICE Trans. Commun., vol.E88-B, no.1, pp.10-18, Jan. 2005.

[25] A. Chini, Y. Wu, M. El-Tanany, and S. Mahmoud, "Filtered decision feedback channel estimation for OFDM-based DTV terrestrial broadcasting system," IEEE Trans. Broadcast., vol.44, no.1, pp.211, March 1998.

[26] T. Fukuhara, H. Yuan, Y. Takeuchi, and H. Kobayashi, "A novel channel estimation method for OFDM transmission technique under fast time-variant fading channel," Proc. IEEE VTC2003-Spring, vol.4, pp.2343-2347, April 2003.

[27] K. Ishihara, K. Takeda, and F. Adachi, "Pilot-assisted decision feedback channel estimation for STTD OFDM mobile radio," IEICE Trans. Commun., vol.E88-B, no.2, pp.561-567, Feb. 2005.

[28] J. Choi and R.W. Heath, Jr., "Interpolation based transmit beamforming for MIMO-OFDM with limited feedback," IEEE Trans. Signal Process., vol.53, no.11, pp.4125-4135, Nov. 2005.

[29] K. Tsunekawa, K. Nishimori, R. Kudo, Y. Takatori, and N. Tachikawa, "Beamforming and antenna design techniques for realizing wideband MIMO-OFDM system," Proc. IEEE AP-S Intl. Symp., vol.2A, pp.329-332, July 2005.

[30] Q. Li and X.E. Lin, "Compact feedback for MIMO-OFDM systems over frequency selective channels," Proc. IEEE VTC2005-Spring, vol.1, pp.187-191, May/June 2005.

[31] B.H. Phu, Y. Ogawa, T. Ohgane, and T. Nishimura, "Channel extrapolation techniques for E-SDM systems in time-varying fading environments," IEICE Trans. Commun., vol.E89-B, no.11, pp.30833092, Nov. 2006

[32] H. Matsuoka, S. Sampei, N. Morinaga, and Y. Kamio, "Adaptive modulation system with punctured convolutional code for high quality personal communication systems," IEICE Trans. Commun., vol.E79-B, no.3, pp.328-334, March 1996.

[33] T. Ohgane, T. Nishimura, and Y. Ogawa, "Applications of space division multiplexing and those performance in a MIMO channel," IEICE Trans. Commun., vol.E88-B, no.5, pp.1843-1851, May 2005.

[34] J.G. Proakis, Digital Communications, fourth ed., McGraw-Hill, New York, 2000

\section{Appendix: On Effects of Ordering in GS Processing}

As stated in Sect. 3.2, vector norm-based ordering in GS processing is effective to obtain vectors similar to TEVs. However, since the order changes at some frequency points in a frequency selective fading environment, such ordering may cause frequency discontinuity of the vectors and effective channels. In this Appendix, we examine and prove these effects of ordering. For the sake of convenience, hereinafter we refer to the PEV scheme using GS operation with normbased ordering as PEV with norm-ordered GS.

Figure A. 1 illustrates the example of pseudo eigenvalues of $4 \times 4$ MIMO obtained by PEVs with norm-ordered GS in Scenario B, where the delay profile of the multipath channel is the same as one in Fig. 3(b). Compared with the conventional PEV case shown in Fig. 3(b), PEVs with normordered GS provide pseudo eigenvalues closer to true eigenvalues. We can say that the ordering is effective to obtain better weight vectors. However, it is seen that frequency discontinuity points occur due to the ordering. Let us discuss the impact of the discontinuity on delay spreads in the following.

Figure A. 2 shows mean power delay profiles of effective channels obtained by PEVs with norm-ordered GS in a $4 \times 4$ MIMO system. Here, Figs. A.2(a) and (b) are comparable to Figs. 2(a), (b) and (c), (d) because of the same setups and scenarios, respectively. It is obvious that in both scenarios the ordering causes larger delay spreads compared 


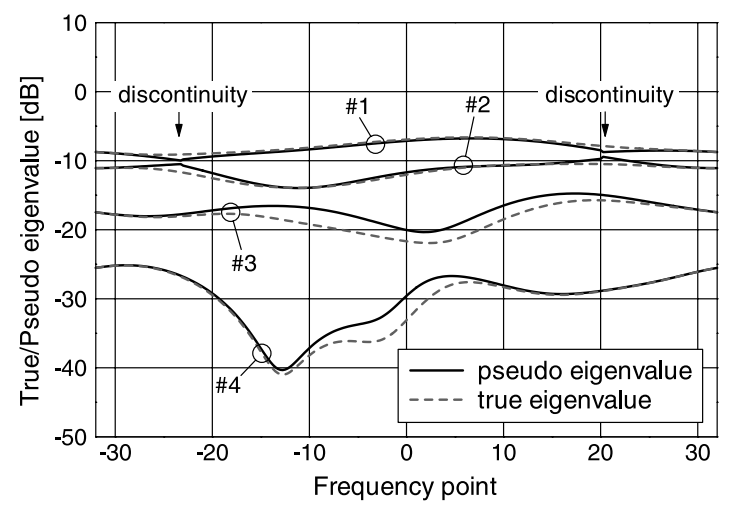

Fig. A. 1 Example of pseudo eigenvalues of $4 \times 4$ MIMO obtained by PEVs with norm-ordered GS in Scenario B.

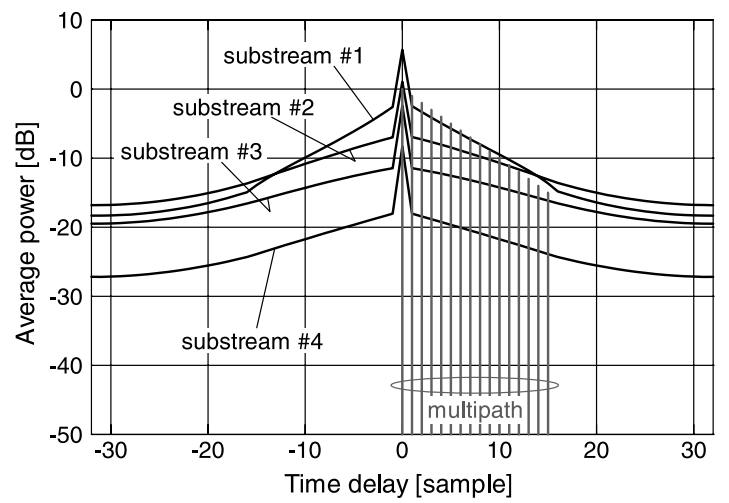

(a) Scenario A

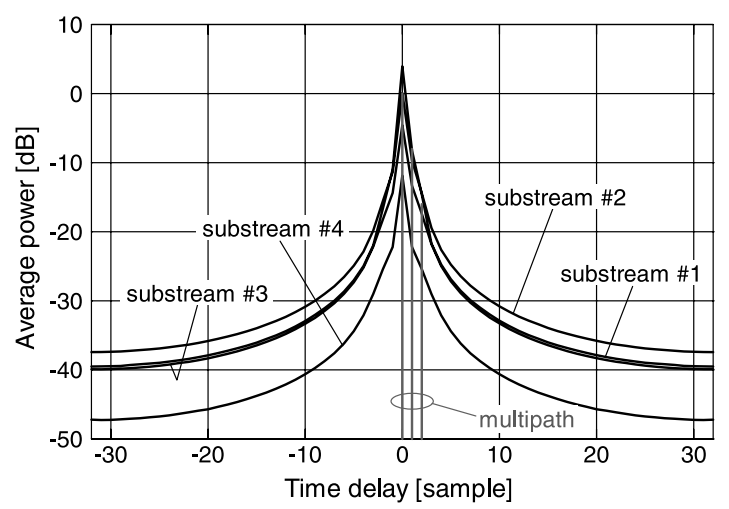

(b) Scenario B

Fig. A.2 Mean power delay profiles of effective channels in a $4 \times$ 4 MIMO case of PEV with norm-ordered GS.

with the conventional PEV cases shown in Figs. 2(a) and (c). Consequently, while ordering is an effective solution to calculate weight vectors closer to TEVs, it may cause frequency discontinuity and enlarge delay spreads of effective channels. In our study, therefore, we do not employ such ordering in PEV calculation.

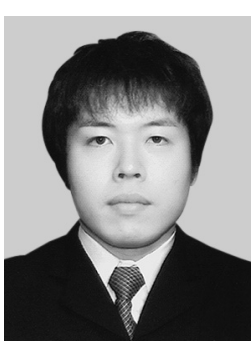

Hiroshi Nishimoto received the B.E. and M.E. degrees from Hokkaido University, Sapporo, Japan, in 2003 and 2005, respectively. He is currently working toward the Ph.D. degree at the Graduate School of Information Science and Technology, Hokkaido University. His research interests are in MIMO propagation measurement and MIMO communication systems. He has been a Research Fellow of the Japan Society for the Promotion of Science since 2005. He received the IEEE VTS Japan Chapter Student Paper Award in 2007, the Young Researchers' Award of IEICE Japan in 2007, and the 2006 IEICE RCS Active Research Award. He is a student member of the IEEE.

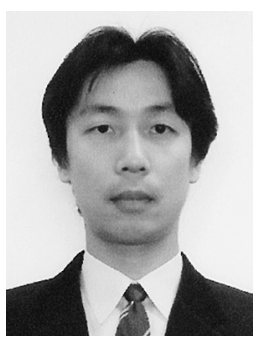

Toshihiko Nishimura received the B.S. and M.S. degrees in physics and Ph.D. degree in electronics engineering from Hokkaido University, Sapporo, Japan, in 1992, 1994, and 1997, respectively. In 1998, he joined the Graduate School of Engineering (reorganized to Graduate School of Information Science and Technology at present) at Hokkaido University, where he is currently an Assistant Professor of the Graduate School of Information Science and Technology. His current research interests are in MIMO systems using smart antenna techniques. Dr. Nishimura received the Young Researchers' Award of IEICE Japan in 2000, and the Best Paper Award from IEICE Japan in 2007. Dr. Nishimura is a member of the IEEE.

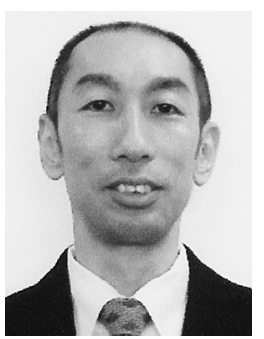

Takeo Ohgane received the B.E., M.E., and $\mathrm{Ph} . \mathrm{D}$. degrees in electronics engineering from Hokkaido University, Sapporo, Japan, in 1984, 1986, and 1994, respectively. From 1986 to 1992, he was with Communications Research Laboratory, Ministry of Posts and Telecommunications. From 1992 to 1995, he was on assignment at ATR Optical and Radio Communications Research Laboratory. Since 1995, he has been with Hokkaido University, where he is an Associate Professor. During 2005-2006, he was at Centre for Communications Research, University of Bristol, U.K., as a Visiting Fellow. His interests are in MIMO signal processing for wireless communications. Dr. Ohgane received the IEEE AP-S Tokyo Chapter Young Engineer Award in 1993, the Young Researchers' Award of IEICE Japan in 1990, and the Best Paper Award from IEICE Japan in 2007. Dr. Ohgane is a member of the IEEE.

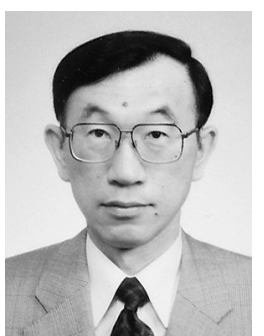

Yasutaka Ogawa received the B.E., M.E and Ph.D. degrees from Hokkaido University, Sapporo, Japan, in 1973, 1975, and 1978, respectively. Since 1979, he has been with Hokkaido University, where he is currently a Professor of the Graduate School of Information Science and Technology. During 1992-1993, he was with ElectroScience Laboratory, the Ohio State University, U.S.A., as a Visiting Scholar, on leave from Hokkaido University. His interests are in adaptive antennas, mobile communications, super-resolution techniques, and MIMO systems. Dr. Ogawa received the Yasujiro Niwa outstanding paper award in 1978, the Young Researchers' Award of IEICE Japan in 1982, and the Best Paper Award from IEICE Japan in 2007. Dr. Ogawa is a senior member of the IEEE. 